\title{
Knee Android Model Reproducing Internal-External Rotation with Screw-Home Movement of the Human Knee
}

\author{
Daichi Yamauchi, Sho Takei, Noritaka Sato, Yoshifumi Morita \\ Department of Computer Science and Engineering, Nagoya Institute of Technology, \\ Gokiso-cho, Showa-ku, Nagoya, Aichi 466-8555, Japan \\ E-mail:d.yamauchi.170@nitech.ac.jp
}

\begin{abstract}
This paper proposes a knee android model (Knee-AM) that can reproduce the internal-external rotation with screwhome movement (SHM) of the human knee. This rotation of the Knee-AM was realized by changing the number, lengths, and fixing points of the nylon-cords of our previous Knee-AM. Moreover, when the anterior cruciate ligament (ACL) was removed from the Knee-AM to imitate ligament injury, SHM did not occur during knee extension. The results show the important role played by the ACL in SHM.
\end{abstract}

Keywords: Android model, Knee model, Human knee joint, Screw-home movement, Joint mechanism.

\section{Introduction}

In the recent times, the time available for rehabilitation treatment is limited and the number of patients, particularly elderly patients, is increasing. Against this background, efficient and effective treatments are social needs in the field of rehabilitation. As a solution for these needs, we earlier proposed a knee android model (Knee-AM) for supporting treatment planning ${ }^{1}$.

An android model of the human joint was developed by Sanaka to improve the medical technology in the field of rehabilitation ${ }^{2}$. Android models are anatomical models composed of the minimum number of parts required for reproducing the corresponding human movement. They can reproduce the three elements of movement ("slide," "roll," and "leave") that occur between two articulating bones. Sanaka ${ }^{2}$ found that the kinematic mechanisms of an android model of the index finger had some different properties from those of the actual movement of the index finger. Moreover, rehabilitation based on the knowledge gained from android models has contributed to improving the range of motion, which was reduced upon tenorrhaphy. Consequently, Sanaka's study confirmed that the knowledge obtained using android models is beneficial for medical treatment.

The internal-external (IE) rotation during knee extension in humans can be classified into three types ${ }^{3}$, namely, "External rotation type," "Internal rotation at the end type," and "Internal rotation type". In our previous study, we proposed a Knee-AM consisting of a tibial model, a femoral model, and 10 nylon-cords imitating the ligaments. The IE rotation of the KneeAM was similar to the internal rotation at the end type. This type did not occur commonly, and often occurred in humans with joint laxity ${ }^{3}$.

In this study, we aimed to develop a Knee-AM that can reproduce the IE rotation of the external rotation type. Since most of the human knee motion belongs to this type, reproducing this type in the Knee-AM is important for using the model on a wider scale. Additionally, since external rotation at the end of extension, that is, the screw-home movement (SHM), in this type, we investigated the mechanism of the SHM using the Knee-AM. Furthermore, we also aimed to gain knowledge of a ligament injury by simulating the injury in the Knee-AM. 


\section{Knee Android Model (Knee-AM)}

The Knee-AM is shown in Fig. 1. It imitates the right knee joint and consists of a tibial model, a femoral model, and five nylon-cords. To allow the Knee-AM extend automatically, we used a drive unit.

\subsection{Bone models}

We used the same bone models as those in our previous study $^{1}$. The tibial and the femoral models, imitating the tibia and the femur, were made of a smooth nylon-based material by using a 3D printer. The femoral model was cut near the knee joint to about one-fifth of its full length. An aluminum rod was attached to the cut surface along the long-axis of the femoral model to fix the femoral model to the drive unit. A lightweight square plate (length of side: $150 \mathrm{~mm}$ ) also was attached to the tibial model for attaching measurement markers.

\subsection{Nylon-cords}

We used five inextensible nylon-cords to imitate the ligaments of the knee on the basis of the motion and the anatomy of the human $\mathrm{knee}^{4}$, as shown in Fig. 2. The nylon-cords were attached to the bone models by copper nails. When measuring the lengths of the nylon-cords, we measured the lengths between the centers of two copper nail heads. The correspondences of the five nylon-cords to the ligaments of the human knee, and the lengths of these cords are listed in Table 1. We used fewer nylon-cords than in our previous Knee-AM ${ }^{1}$ to investigate knee motion in a simple structure; we also rearranged the positions and the lengths of the nyloncords to reproduce the human knee motion precisely.

The tibial collateral ligament (TCL) was imitated by one nylon-cord (Cord T). One end of Cord $\mathrm{T}$ was

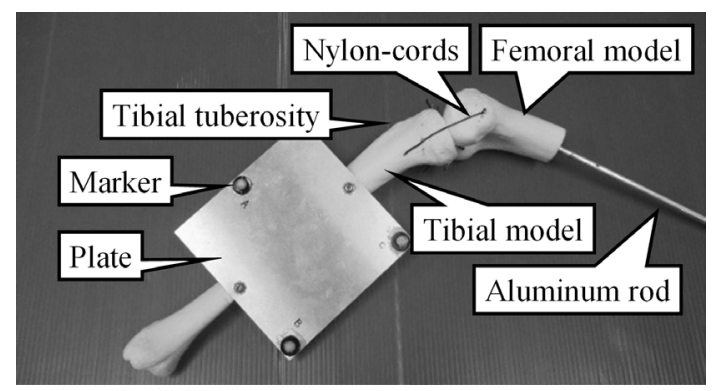

Fig. 1. Knee android model (Knee-AM) attached near the center of the medial femoral condyle (MFC) so that Cord T did not become loose when the flexion angle changed from $90^{\circ}$ to $0^{\circ}$. The other end was attached to the posterior of the insert part of the pes anserinus.

The fibular collateral ligament (FCL) was imitated by one nylon-cord (Cord F). One end of Cord F was also attached near the center of lateral femoral condyle (LFC) so that Cord F did not become loose when the flexion angle changed from $90^{\circ}$ to $0^{\circ}$. The other end in the actual knee is attached to the fibular head. However, since the Knee-AM did not include the fibular model, the other end was attached to the insert part of the posterior ligament of the fibular head.

The anterior cruciate ligament (ACL) was imitated by two nylon-cords (Cords A1 and A2, collectively referred to as Cord A). Two nylon-cords were used because the ACL and the PCL consist of many fibers of different lengths ${ }^{4}$. One end of Cord A1 was attached to the distal posterior of the LFC. The other end was attached near the insert part of the medial meniscus. Similarly, one end of Cord A2 was attached to the proximal posterior of the LFC. The other end was attached at the medial posterior $9 \mathrm{~mm}$ from the end of Cord A1 on the tibial model.

The posterior cruciate ligament (PCL) was imitated by one nylon-cord (Cord P). In order to reproduce the motion between a flexion angle of $90^{\circ}$ and full extension, we imitated the PCL, which tightens at $90^{\circ}$ of flexion $^{4}$, with one nylon-cord. One end of Cord $\mathrm{P}$ was attached to the distal anterior of the MFC. The other end was attached to the center posterior of the tibial plateau.

Table 1. Lengths and correspondences of the five nylon-cords used in the model to the ligaments of the human knee (TCL: Tibial collateral ligament, FCL: Fibular collateral ligament, ACL: Anterior cruciate ligament, PCL: Posterior cruciate ligament)

\begin{tabular}{ccr}
\hline Name of cord & Ligament & Length $[\mathrm{mm}]$ \\
\hline Cord T & TCL & 58.5 \\
Cord F & FCL & 39.3 \\
Cord A1 & ACL & 34.2 \\
Cord A2 & ACL & 37.3 \\
Cord P & PCL & 31.1 \\
\hline
\end{tabular}




\subsection{Drive unit}

The drive unit and the Knee-AM are shown in Fig. 3. The drive unit comprised a motor, a reel fixed on the motor shaft, a pulley, a wire, and a frame. The drive unit extended the joint of the Knee-AM by pulling a wire connected to the tibial tuberosity. The wire was run through the pulleys and wound onto the reel. The motor was placed above the long-axis of the femoral model so that the wire made an angle of $0^{\circ}$ horizontally with respect to the long-axis of the femoral model. The pulley was placed at a position corresponding to the human patella at full extension. The femoral model was fixed on the frame by clamping the aluminum rod and the proximal part of the femoral model. This fixing method is an improvement of that used in our previous study $^{1}$ to fix the Knee-AM on the drive unit firmly.

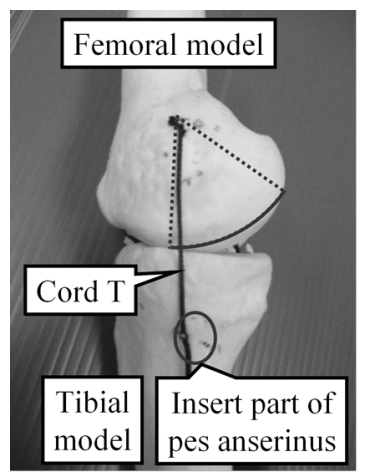

(a) Tibial collateral ligament (TCL)

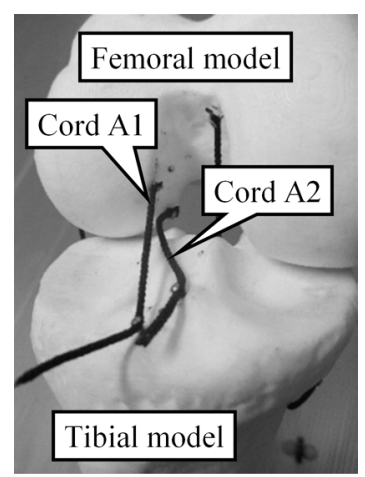

(c) Anterior cruciate ligament (ACL)

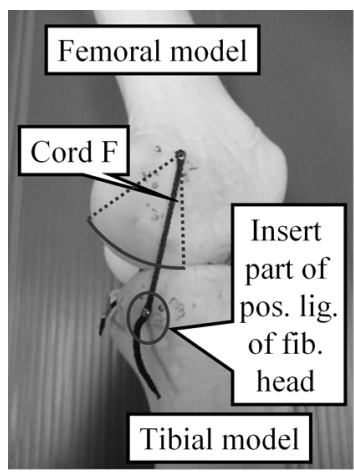

(b) Fibular collateral ligament (FCL)

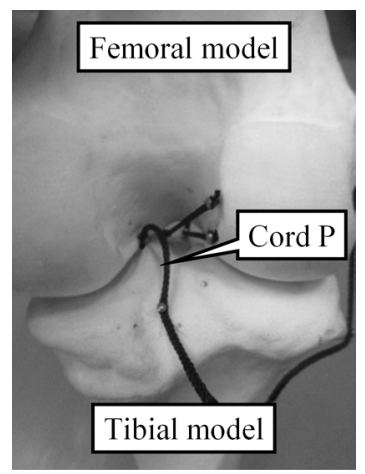
ligament (PCL) (d) Posterior cruciate

Fig. 2. Nylon-cords imitating the ligaments in the human knee

\section{Experiment}

We conducted two experiments to confirm the utility of the Knee-AM. In the experiments, we measured the motions of the bone models with a motion capture system VICON (Vicon Movement Systems Ltd.) and calculated the flexion angle and IE rotation angle by using the same calculation methods as in our previous study ${ }^{1}$.

\subsection{Experiment method}

First, we tested the reproducibility of the Knee-AM for the IE rotation of the human knee. In this test, the KneeAM was extended from $90^{\circ}$ of extension angle to full extension by the drive unit. To evaluate the reproducibility, we compared the IE rotation of KneeAM with that of the human knee.

Second, we investigated the knee motion of an ACL injury by using the Knee-AM. In this simulation, Cords A1 and A2 were removed, as shown in Fig. 4. The Knee-AM simulating an ACL injury is referred to as the injured Knee-AM. The experimental procedure was the same as that of the first experiment.

The measured data included small vibrations caused by the mechanical resonance of the Knee-AM. In order to eliminate these vibrations, we used a low-pass filter with a cutoff frequency of $5 \mathrm{~Hz}$.

\subsection{Results}

The IE rotations of the Knee-AM and the injured KneeAM are shown in Fig. 5. In order to compare the IE rotations of these two types of Knee-AMs with those of the human knee, we used the human knee movement data obtained from Ref. 3.

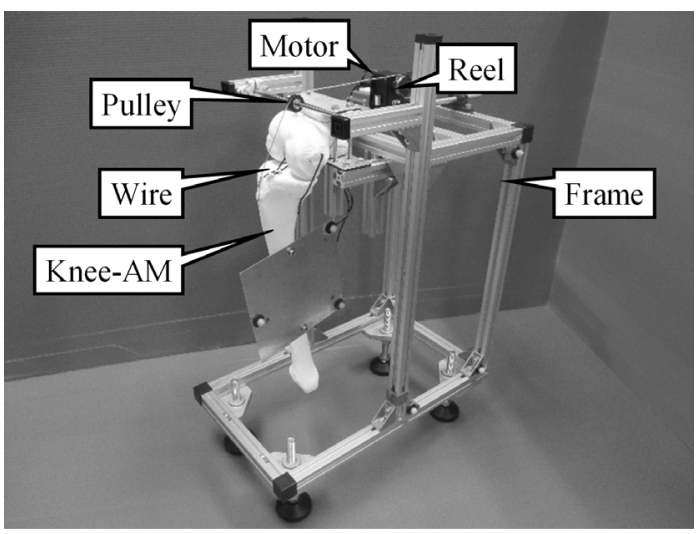

Fig. 3. Drive unit and Knee-AM 
In the graph in Fig. 5, the horizontal axis is the flexion angle, with extension denoted in the positive direction. The vertical axis is the IE rotation angle, with internal rotation denoted in the positive direction. The rotation angle is set to be zero at a flexion angle of $-90^{\circ}$. The markers show the maximum absolute value of the rotation angle between flexion angles of $-90^{\circ}$ and $0^{\circ}$.

Ishii and Yamamoto ${ }^{3}$ classified IE rotation of 30 subjects into three types. "Type 1" corresponded to the external rotation at the end of extension, namely, SHM. "Type 2" corresponded to external rotation occurring at the beginning of extension, and internal rotation occurring in the second half of extension. "Type 3" corresponded to internal rotation occurring over the entire extension.

As seen from the figure, in the case of Knee-AM, the round marker corresponded to Type 1; that is, SHM occurred. Therefore, the Knee-AM can reproduce the IE rotation with SHM of the human knee. On the other hand, in the injured Knee-AM, the round marker corresponded to Type 1, though SHM did not occur. Thus, it is inferred that the ACL is a necessary component for SHM.

\subsection{Discussion}

We considered the mechanism causing SHM. Comparison of the waveforms of the Knee-AM and the injured Knee-AM in Fig. 5 show differences in movements between flexion angles of $-20^{\circ}$ and $0^{\circ}$. Accordingly, it was assumed that Cord A started to tighten from $-20^{\circ}$ and that the SHM was caused by Cord A.

In order to clarify the mechanism causing the SHM, we observed the motions of the lateral and the medial plateaus with respect to the femoral model and the tensions of the nylon-cords of the two types of Knee-

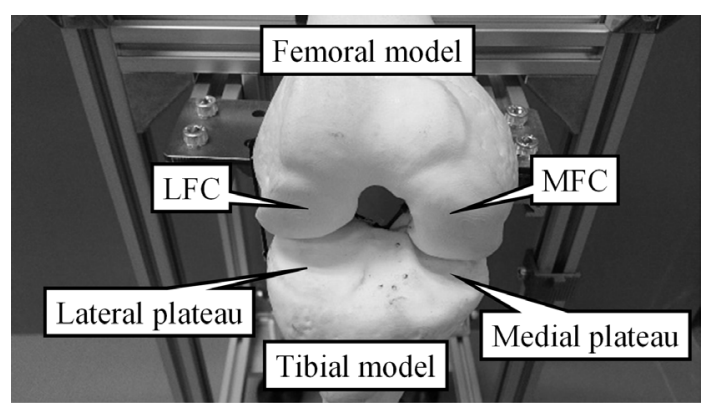

Fig. 4. Injured Knee-AM
AMs; these data are listed in Table 2. In the case of the Knee-AM, Cords T, F, and P were weakly stretched at a flexion angle of $-90^{\circ}$. When the Knee-AM started to extend, the lateral and the medial plateaus rolled from a flexion angle of $-90^{\circ}$ to $-60^{\circ}$, and then these plateaus started to slide toward the anterior. In addition, the tension in Cord $\mathrm{T}$ increased until full extension, and Cord $\mathrm{P}$ became loose. Around a flexion angle of $-20^{\circ}$, Cord A started to tighten, and the lateral plateau ceased to slide and began to roll. In contrast, the medial plateau kept sliding toward the anterior. The differences in the motions of the lateral and the medial plateaus cause $\mathrm{SHM}^{4}$. Additionally, Cord $\mathrm{F}$ tightened with the roll of the lateral plateau. At full extension, Cords T, F, and A tightened strongly, and the tibial model became fixed on the femoral model. This means that the phenomenon in which the knee was locked occurred in the Knee-AM.

The above-described motion of the Knee-AM was in agreement with that described in Ref. 4 with regard the following points:

(i) the lateral plateau rolls to a greater extent than the medial plateau,

(ii) the TCL and the FCL tighten during extension, and

(iii) the ACL tightens at the extended position, and the PCL tightens at the flexed position.

This agreement implies that the knowledge gained using the Knee-AM is valid.

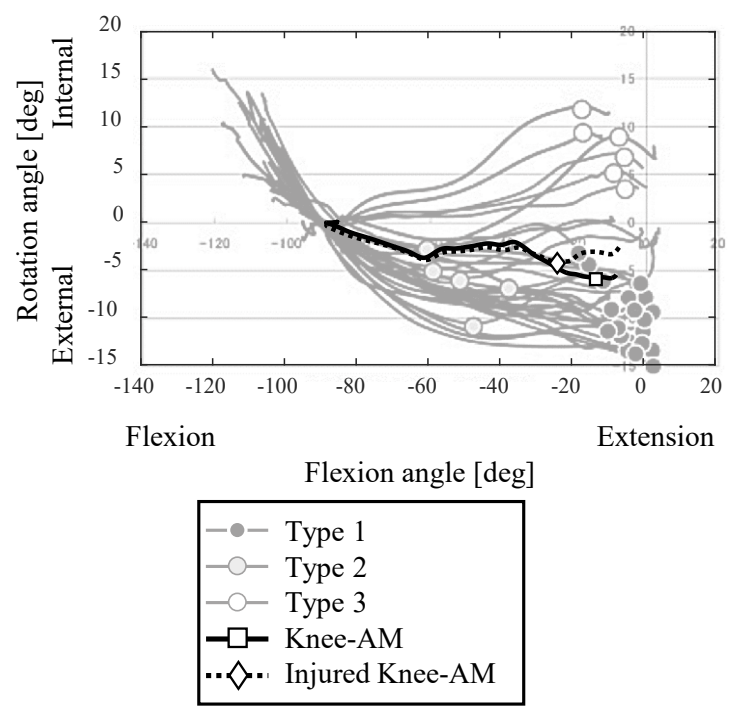

Fig. 5. Comparison of internal-external rotations during extension of Knee-AM and thirty human subjects ${ }^{3}$. 
On the other hand, in the case of the injured Knee$\mathrm{AM}$, the motion of lateral plateau and the tension in Cord $\mathrm{F}$ were different from those of the Knee-AM between flexion angles of $-20^{\circ}$ and $0^{\circ}$. The lateral plateau continued sliding toward the anterior until the full extension, but Cord $\mathrm{F}$ did not strongly tighten. Therefore, SHM did not occur, and the tibial model did not become fixed on the femoral model at full extension. This means that the phenomenon which the knee was locked did not occur in the injured Knee-AM.

Table 2. Time chart of the tension in the nylon-cords and the motion of the medial and lateral plateaus (WT: Weakly Tight, T: Tight, ST, Strongly Tight, L: Loose, S: Slide, R: Roll)

(a) Knee-AM

\begin{tabular}{c|ccccc}
\hline Flexion angle $\left[^{\circ}\right]$ & -90 & & -20 & & 0 \\
\hline Tension of Cord T & WT & $\nearrow$ & T & $\nearrow$ & ST \\
Tension of Cord F & WT & $\rightarrow$ & WT & $\nearrow$ & ST \\
Tension of Cord A & L & $\rightarrow$ & L & $\nearrow$ & ST \\
Tension of Cord P & WT & $\searrow$ & $\mathrm{L}$ & $\rightarrow$ & $\mathrm{L}$ \\
\hline Motion of medial plateau & $\mathrm{R}$ & $\mathrm{S}$ & $\mathrm{S}$ & $\mathrm{S}$ & $\mathrm{R}$ \\
Motion of lateral plateau & $\mathrm{R}$ & $\mathrm{S}$ & $\mathrm{S}$ & $\mathrm{R}$ & $\mathrm{R}$ \\
\hline
\end{tabular}

(b) Injured Knee-AM

\begin{tabular}{c|ccccc}
\hline Flexion angle $\left[^{\circ}\right]$ & -90 & & -20 & & 0 \\
\hline Tension of Cord T & WT & $\nearrow$ & T & $\nearrow$ & T \\
Tension of Cord F & WT & $\rightarrow$ & WT & $\rightarrow$ & WT \\
Tension of Cord A & & & None & & \\
Tension of Cord P & WT & $\searrow$ & L & $\rightarrow$ & L \\
\hline Motion of medial plateau & $\mathrm{R}$ & $\mathrm{S}$ & $\mathrm{S}$ & $\mathrm{S}$ & $\mathrm{S}$ \\
Motion of lateral plateau & $\mathrm{R}$ & $\mathrm{S}$ & $\mathrm{S}$ & $\mathrm{S}$ & $\mathrm{S}$ \\
\hline
\end{tabular}

\section{Conclusion}

In this paper, we proposed a Knee-AM that can reproduce the IE rotation of external rotation type by rearranging the nylon-cords of our previous Knee-AM. Our investigation of the mechanism of SHM by using the Knee-AM revealed that the roll of the lateral plateau tightens Cord $\mathrm{F}$ and that strong tightening of Cords $\mathrm{T}, \mathrm{F}$, and $\mathrm{A}$ fixes the tibial model on the femoral model. In addition, our simulation results of the ACL injury in the Knee-AM showed that in the absence of the ACL, SHM and the knee-locking phenomenon did not occur.

In future studies, we will experimentally investigate the roles of other ligaments. We also plan to discuss our findings with medical personnel with regard the medical treatments, the improvement in medical technology, and other applications of the Knee-AM.

\section{References}

1. D. Yamauchi, N. Sato, and Y. Morita, An Experimental Study on the Relationship between the Components and Movement of the Human Knee Using an Android Model - Measurement of Internal-External Rotation and Anterior-Posterior Tibial Translation -, in Procs. of Int. Conf. on Control, Automation and Systems 2015 (BEXCO, Busan, 2015), pp. 811-815.

2. K. Sanaka, Android with Biological Structure for Supporting Medical Technology, in Tokai-Section Joint Conference on Electrical, Electronics, and Related Engineering (Shizuoka Univ., Hamamatsu, 2013), S2-5. (in Japanese)

3. S. Ishii and S. Yamamoto, Kinematic Analysis of Screw Home Motion with Active Knee Extension in the NonWeight Bearing Position, in Rigakuryoho Kagaku, 23(1) (2008), pp. 11-16. (in Japanese)

4. I. A. Kapandji and E. Shiota (trasl.), Physiology of the Joints: Volume 2 Lower Limb, 6th edn. (Ishiyaku Publishers, Inc., Tokyo, 2010), pp. 108-129. (in Japanese) 